\title{
Comments on: Extensions of some classical methods in change point analysis
}

\author{
John A. D. Aston
}

Published online: 9 May 2014

(C) Sociedad de Estadística e Investigación Operativa 2014

I would firstly like to congratulate the authors on such a thorough and well-written survey of some of the recent exciting extensions in change point analysis from classical methods. The authors have clearly demonstrated that this is a rapidly evolving area, which has both interesting theoretical challenges and modern and complex applications for which such methodology is required.

I would like to concentrate this discussion on two particular interrelated issues which were not covered in such detail as some of the other areas in the discussion; power analysis of change point methods particularly when using dimension reduction and applications to complex data, such as brain imaging data, where model misspecification might well be an issue. Indeed, these could actually both be considered as one issue, in that dimension reduction (assuming that the true dimension is larger than the reduced space) is in some sense also model misspecification.

While dimension reduction techniques are applicable to multivariate and panel data, it is functional data which will be used to motivate the discussion (see Section 7 in the paper). The approach in Berkes et al. (2009) defined a CUSUM process based on reducing the dimension of the functional data using a small (finite, say $d$ ) number of bases functions determined from principal component analysis and then treating the resulting projections in an analogous way to multivariate data. This, of course, is sensible in that it can yield a limiting distribution under reasonable assumptions. However, the normalisation by the variance (the estimated eigenvalue) inherently increases the prominence of the small eigenfunctions (which are likely to be simply noise) in relation to the more dominant eigendirections. This increases the misspecification of the model

This comment refers to the invited paper available at doi:10.1007/s11749-014-0368-4.

\footnotetext{
J. A. D. Aston ( $\varangle)$

Statistical Laboratory, DPMMS, University of Cambridge, CB3 0WB Cambridge, UK e-mail: J.Aston@statslab.cam.ac.uk
} 
to the original functional specification, although in the limit, this is unlikely to be of consequence for non-vanishing changes. However, more importantly, any change that happens in a space that is orthogonal to the space spanned by these $d$ eigenfunctions, cannot be detected. It is therefore crucial to ascertain that any reasonable direction for a change (where the direction is specified by $\delta(t)$ of Section 7 ) is not orthogonal to a dimension reduction chosen. Indeed, this is inherently related to the power of the test, as a change orthogonal to the $d$ eigenfunctions has no power, while a change that is not orthogonal has (finite sample) power related to the size of the change in the projected space, which will depend on both the eigendirection and the normalisation. However, the use of principal components as a choice of dimension reduction can certainly be of use in this regard. As shown by Aston and Kirch (2012), using an eigendecomposition of the estimated covariance can be superior even if the true uncontaminated covariance is known, as it is possible to detect changes in this situation which cannot be detected if an eigendecomposition of the true uncontaminated covariance is used.

The issue of model misspecification or approximation is very much related. In many instances, particularly for so-called "big data", the model for the change (be it a change in mean, variance or autocovariance structure) is a crude approximation to the true change in the data generation process (for example, a gradual change in the mean might be approximated by a step change), while specifying a completely free distributional assumption [such as in Csörgo and Horváth (1987)] may not be feasible to compute. It is therefore likely that in addition to trying to characterise the power properties of change point detection algorithms under the alternative, it will become increasingly of interest to characterise the properties under a range of alternatives, not simply the one specified by the model. In most cases, if the procedure has asymptotic power one against the alternative, and the alternative investigated is not orthogonal to the true change process, then the procedure will also have asymptotic power one in this case as well. However, more relevant for many modern applications are the finite sample and small, contiguous, alternative settings.

\section{References}

Berkes I, Gabrys R, Horváth L, Kokoszka P (2009) Detecting changes in the mean of functional observations. J R Stat Soc Ser B Stat Methodol 71:927-946

Aston JAD, Kirch C (2012) Detecting and estimating changes in dependent functional data. J Multivar Anal 109:204-220

Csörgo M, Horváth L (1987) Nonparametric tests for the changepoint problem. J Stat Plan Inference 17:1-9 